\section{Pure gold}

Lysosomes in Biology and Pathology, Volume 4. (North-Holland Research Monographs: Frontiers of Biology, Volume 43.) Edited by J. T. Dingle and R. T. Dean. Pp. xviii +614 . (North-Holland: Amsterdam and Oxford; American Elsevier: New York, 1975.) $\$ 74.95$; Dfl. 180.00 .

THE 'gold rush' following the discovery of lysosomes has now subsided. The easily accessible nuggets have been removed and it is now necessary to dig more deeply and systematically to reap rewards. That they are still there is illustrated by several chapters in this volume, which report thorough analyses of old problems, such as the role of lysosomes in degradation of plasma proteins (G. Gregoriadis) and in intracellular protein turnover (H. L. Segal). Several chapters are devoted to lysosomes in different cell types (skeletal and cardiac muscle cells, blood platelets, arterial walls, skin, Tetrahymena) but these accounts are more than descriptive, embracing the participation of lyso-

\section{Starch structure}

Starch and its Components. By W. Banks and C. T. Greenwood. Pp. $x i+342$. (Edinburgh University: Edinburgh, 1975.) £10.00.

STARCH, particularly as it occurs in cereal grains, is the major carbohydrate food ingredient in the human diet. Yet the structure of the starch granule is still unknown and no one has synthesised a granule in vitro. Nevertheless a great amount is known about starch granules and particularly about the structure, behaviour and synthesis of its major molecular components, amylose and amylopectin.

This book reviews the chemical nature of starch and its components, and their behaviour with enzymes. The book is a highly biased presentation and as the authors state in the Preface "We make no apology for the fact that emphasis in this monograph is on our own work..." Not everyone will agree with all the authors' statements in the book, but I find the total coverage good and the many arguments and criticisms sound. A reader will carry away a fine sense of starch bchaviour and a good feel for the structure and reactions of amylose and amylopectin. Emphasis is placed on hydrodynamic behaviour and on the action of enzymes on amylose and amylopectin.

After a short introduction the book is separated into five major sections. The first deals with the fractionation of starch and the fine structure of its somes in the physiology and pathology of the cells concerned. For example, blood platelets are rich in lysosomes, and the release of their enzymes contributes to the pathogenesis of inflammation; and storage of lipids in lysosomes of cells in arterial walls may be important in atherosclerosis.

Evidence is presented of heterogeneity of lysosomes within single cell types (M. Davies) and of multiple forms of lysosomal enzymes (R. T. Dean). Speculative chapters include those of C. M. Szego on lysosomes in nucleocytoplasmic communication and L. J. Ignarro on the regulation of lysosomal enzyme secretion. In these days when relevance is so much emphasised it is pleasing to see a beautifully illustrated account by Yolande Heslop-Harrison of enzyme rclease in carnivorous plants. As in previous volumes, the production is of high quality, with many illustrations, including well-reproduced half tones, and comprehensive author and subject indices. The quality of production is unfortunately reflected in the price.

A. C. Allison

components. Several fractionation procedures are discussed and the chemical but mainly enzyme reactions of components are given. Emphasis is placed on the use of enzymes to elucidate starch molecular structure. A section of 43 pages is devoted to the reaction of starch and its components with iodine. A section concerned with the conformation of amylose in solution provides a wide coverage of methods for conformational determination and possible interpretations. It is agreed that the basic helical nature of amylose predominates in its complexes and is lowest, if existant at all, in neutral aqueous solution. Starch-degrading enzymes are treated by examining the action pattern of exo-, endo- and branching or debranching enzymes. Pitfalls can be numerous in the use of enzymes to deduce starch structure and many of these are clearly indicated. The final major section carries a discussion of the structure and biosynthesis of the starch granule. Attention is directed toward crystalline nature and orientation.

This is not a practical book. It does not discuss the characteristics of starch pastes nor of the preparation or use of starch or its modifications. The book is devoted to a discussion of the basic structure of starch and its components as interpreted by the authors. As such it is recommended to all those having an interest in the fundamental characteristics of starch. Biochemists will find the review useful and interesting. Roy L. Whistler

\section{Amateur astronomers' Bible}

Astronomy: A Handbook. Edited by G. D. Roth. Translated and Revised by Arthur Beer. Pp. xviii +567. (Springer: Berlin and New York, 1975.) DM44.90; $\$ 18.50$.

THIS is a revised and translated version of what was for long the German amateur astronomers' bible, Handbïch für Sternfreunde, described rather misleadingly on the dust jacket as "designed to fill the needs of both professional and amateur astronomers". It in fact sets out to encourage the amateur to make and reduce observations of professional quality and rigour, so that the two might be complementary.

The ground covered is naturally similar to that in other amateurs' handbooks such as J. B. Sidgwick's $O b$ servational Astronomy for Amateurs and Amateur Astronomer's Handbook, but its approach is more sophisticated. The chapter on 'Applied Mathematics for Amateur Astronomers' requires maths of at least A-level standard, and several expressions elsewhere in the book would be clarified by the use of worked examples. Yet the handbook is curiously out of date in parts; the radio astronomy chapter contains circuit diagrams which abound in valves, and some of the definitions in this and other chapters have a ring of history about them. There are some strange omissions too, in what is overall a very comprehensive text. Narrow band and even white light observations of solar flares are not discussed; meteor triangulation is dismissed in one sentence, and meteor spectroscopy, where the amateur can contribute important results, is not even mentioned.

There are, however, some particularly good chapters which are not covered well elsewhere. 'Fundamentals of Spherical Astronomy' is an excellent compact reference to position and time measurements. The chapters on solar eclipses, lunar eclipses and artificial Earth satellites are also most informative, and that on 'Observation of the Planets' stresses the amateur's role in continuously monitoring changes in planetary detail. There is a useful and complete appendix; and although several of the original references in the text are necessarily in German, the very thorough bibliography suggests much in the way of alternative reading.

Although this book is unlikely to attain the popular appeal of Sidgwick's handbooks, it is a good reference work for the advanced amateur astronomer and will be welcomed by many.

Heather A. Couper 